\title{
A mechanosensitive vascular niche for Drosophila hematopoiesis
}

michele Crozatier ( $\nabla$ michele.crozatier-borde@univ-tlse3.fr )

CBI, MCD https://orcid.org/0000-0001-9911-462X

\section{Yushun Tian}

CBI, MCD

Ismaël Morin-Poulard

Centre de Biologie du Développement

Nathalie Vanzo

Centre de Biologie du Développement

\section{Article}

Keywords:

Posted Date: February 22nd, 2022

DOI: https://doi.org/10.21203/rs.3.rs-1326573/v1

License: (c) (i) This work is licensed under a Creative Commons Attribution 4.0 International License. Read Full License 
1 A mechanosensitive vascular niche for Drosophila hematopoiesis

2

3 Short title: A mechanosensitive hematopoietic niche

4

5

6

One sentence summary: Mechano-sensing of blood flow by vascular niche cells regulates

7 hematopoietic progenitor maintenance

8

9

10

Yushun Tian, Ismaël Morin-Poulard, Nathalie Vanzo and Michèle Crozatier *

11

12

MCD/UMR5077, Centre de Biologie Intégrative (CBI), 118 route de Narbonne 31062 Toulouse

13

Cedex 9, France.

14

15

*To whom correspondence should be addressed: michele.crozatier-borde@univ-tlse3.fr

16

17 


\section{Abstract}

Hematopoietic stem and progenitor cells maintain blood cell homeostasis by integrating various cues provided by specialized microenvironments or niches. Biomechanical forces are emerging as key regulators of hematopoiesis. Here we report that mechanical stimuli provided by blood flow in the vascular niche control Drosophila hematopoiesis. In vascular niche cells, the mechanosensitive channel Piezo transduces mechanical forces through intracellular calcium upregulation, leading to Notch activation and repression of FGF ligand transcription, known to regulate hematopoietic progenitor maintenance. Our results provide insight into how the vascular niche integrates mechanical stimuli to regulate hematopoiesis.

\section{Main text}

In adult Mammals, hematopoiesis takes place in bone marrow where hematopoietic stem and progenitor cells (HSPCs) are maintained by specialized microenvironments, or niches. HSPCs localize around arterioles and sinusoid blood vessels. Endothelium cells, which line the lumen of blood vessels and perivascular cells, constitute the vascular niche $\mathrm{e}^{1-8}$. Endothelium cells are exposed to mechanical forces linked to blood flow ${ }^{9,10}$. Whether mechanosensing by endothelium/vascular niche cells regulates HSPC maintenance is unknown. We use Drosophila as a model system to ask whether mechanical stimulation resulting from blood flow affects the transcription of signals secreted by cardiac cells, which correspond to vascular niche cells in Drosophila, and thereby regulates hematopoietic progenitor maintenance.

The Drosophila larval hematopoietic organ, called the lymph gland, has emerged as an attractive in vivo model to study how hematopoietic progenitor maintenance and differentiation are regulated by their microenvironment ${ }^{11-13}$. The lymph gland is aligned along the cardiac tube which corresponds to the vascular system, and is composed of hematopoietic 
progenitors, differentiated blood cells and the Posterior Signaling Center (PSC). The latter corresponds to the niche (Fig. 1a) since the PSC produces various signals that regulate lymph gland homeostasis, i.e., the balance between progenitors and differentiated blood cells ${ }^{12,13}$. Recently we established that cardiac cells produce the Fibroblast Growth Factor (FGF) ligand Branchless (Bnl). Though the activation of its FGF receptor in progenitors, Bnl controls progenitor maintenance and prevents their differentiation into crystal cells and plasmatocytes, two mature blood cell types that differentiate under physiological conditions. Thus the cardiac tube corresponds to a second niche which directly regulates the extent of progenitor differentiation in the lymph gland ${ }^{14}$. The cardiac tube is composed of one anterior part (aorta) in contact with the lymph gland containing non-contractile cardiomyocytes, and a posterior contractile heart chamber where cardiomyocytes by contracting rhythmically, provide the propelling force for hemolymph/blood flow circulation within the cardiac lumen ${ }^{15}$ (Supplementary Fig. 2a). In insects, the hemolymph provides nutriments and conveys signals, but does not supply oxygen which is achieved via the tracheal system. Analyses of Drosophila cardiac physiology further established that, as in Mammals, the blood flow generates intra-cardiac mechanical forces ${ }^{16}$. Here our data strongly support the conclusion that mechanical constraints exerted by blood flow on vascular niche cells modify their secreted signals and subsequently impact hematopoietic progenitor homeostasis.

\section{Results}

\section{Heartbeat regulates lymph gland homeostasis}

A primary function of Drosophila heart is to pump hemolymph throughout the body in an open circulatory system ${ }^{15}$. We asked whether mechanical constraints exercised by blood flow, resulting from cardioblast contraction, could modify lymph gland hematopoiesis. To stop blood flow, we expressed either a constitutively active form of Ork1 (Ork1-deltaC (Ork1 $\Delta \mathrm{C})$ ), a potassium channel subunit required to set heartbeat frequency ${ }^{17}$ or a knockdown of Myosin 
heavy chain (Mhc, necessary for muscle contraction) specifically in cardiac cells using two independent Gal4 drivers NP1029 and Hand $\Delta \mathrm{C}^{14,18}$. Both Mhc knockdown and Ork1 $\Delta \mathrm{C}$ expression in cardiac cells from the second larval stage (L2) on, resulted in heartbeat arrest (Fig. 1b-c and movies 1-3, Supplementary Fig. 1b) without affecting heart morphology (Supplementary Fig. 1a). In all these contexts, heart failure resulted in decreased cells differentiation in the lymph gland, for both crystal cells (labelled by Hindsight (Hnt) antibody) (Fig. 1e-g, i, Supplementary Fig. 1f-k) and plasmatocytes (P1 antibody labelling) (Supplementary Fig. 1c-e). In agreement with published data ${ }^{17}$ an increased heart rate was observed when Ork1-RNAi was expressed with the Hand $\Delta$ cardiac driver (Fig. 1d, movie 45), and this resulted in increased crystal cell differentiation compared to control (Fig. 1h-i). We then analyzed the effect of heart arrest on hematopoietic progenitors. The transcription factor Collier (Col) (also known as Knot) is expressed at high levels in the PSC niche and at lower levels in core progenitors, a subset of hematopoietic progenitors maintained by cardiac cell signals ${ }^{14,19,20}$ (Fig. 1a). Compared to wild type, higher numbers of $\mathrm{Col}^{+}$progenitors were recorded, indicating that heartbeat controls core progenitor maintenance (Fig. 1j-m,o and Supplementary Fig. 11-n). Since the PSC is also known to control lymph gland homeostasis ${ }^{20-}$ ${ }^{26}$ we looked at PSC cell numbers by Antennapedia (Antp) immunostaining. No difference in PSC cell numbers was observed (Supplementary Fig. 1o-q). Altogether, these data establish that in third instar (L3) larvae the heartbeat, and thereby blood flow, trigger progenitor differentiation into mature blood cells. These results indicate that mechanical stimuli resulting from blood flow may be critical regulators of hematopoiesis.

\section{Mechanosensitive ion channel Piezo controls lymph gland homeostasis}

Heartbeat imposes mechanical constraints on cardiac cells ${ }^{16}$. Piezo encodes a mechanosensitive cation channel that directly senses mechanical tension in lipid bilayers ${ }^{27}$ and is responsible for mechano-reception in many cell types ${ }^{28,29}$. A single piezo gene is 
94

present in the Drosophila genome ${ }^{29-33}$. Piezo is expressed in larval cardiomyocytes, but no function in these cells has been reported so $\mathrm{far}^{29}$. To document its expression, we used piezoGal4, where Gal4 is under the control of a piezo enhancer ${ }^{29}$ and the Piezo knock-in Gal4 allele piezo-Gal4 $(\mathrm{KI})^{30}$ to express UAS-mcd8GFP. In both cases GFP was expressed in cardiac cells and at low levels in a subset of crystal cells (Fig. 1n and Supplementary Fig. 2ac). We investigated whether in cardiac cells Piezo regulates lymph gland hematopoiesis. To monitor blood cell differentiation and progenitor maintenance, we looked at crystal cell differentiation and $\mathrm{Col}^{+}$expression, respectively. In two null mutant contexts: piezo-KO and piezo-Gal4(KI) ${ }^{30}$, crystal cell indexes were lower than in the control (Fig. 1p, $\mathrm{r}$ and Supplementary Fig. 2d-e). Similar results were obtained by knocking down piezo in cardiac cells by RNAi (Fig. 1q, s; Supplementary Fig. 2i-1) or by over-expressing mPiezo1-2336Myc, which encodes a nonfunctional channel ${ }^{31}$ (Supplementary Fig. 2m-o). Higher numbers of $\mathrm{Col}^{+}$progenitors were observed when piezo was knocked down in cardiac cells (Supplementary Fig. 2f-h). Altogether these data establish that piezo expression in cardiac cells regulates lymph gland homeostasis. To determine whether its function is specifically required in anterior aorta cells which are in contact with the lymph gland, we used the 76E11Gal4 cardiac cell driver expressed only in the anterior aorta cells of late L2/early L3 larvae (Supplementary Fig. 2p). Applying piezo knockdown only after the L2 stage using the Gal80 $0^{\text {ts }}$ system $^{34}$, led to a decrease in crystal cell index (Supplementary Fig. 2q-s), which means that piezo is required in anterior aorta cells to control blood cell differentiation. Finally, we showed that piezo knockdown in cardiac cells does not affect heartbeat (Supplementary Fig. $2 \mathrm{t}-\mathrm{u}$ and movie 6). In summary, our data indicate that the function of piezo is required in L3 larval anterior aorta cells to control lymph gland homeostasis non-cell-autonomously. Since piezo knockdown and heart failure led to similar lymph gland defects, we asked whether both were functionally linked. Expression of a constitutively active form of Piezo, mPiezo1-TriM ${ }^{31}$ 
in cardiac cells (Hand $\Delta$ ) led to a significant increase in crystal cell differentiation (Fig. 1t, v). In addition, activation of Piezo function in larvae with reduced heart contractility (Ork1 $\Delta \mathrm{C})$ rescued lymph gland defects as it restored a wild type number of crystal cells (Fig. 1u-v). Overall, these data indicate that in aorta cells piezo acts downstream of heartbeat to control lymph gland hematopoiesis.

\section{Piezo and heartbeat control expression of the FGF ligand Bnl}

Drosophila cardiac cells exert a function similar to the mammalian vascular niche. The FGF ligand Bnl secreted by cardiac cells, activates in L3 larvae the FGF pathway in hematopoietic progenitors, which in turn controls lymph gland homeostasis ${ }^{14}$. To investigate whether Bnl levels in cardiac tube are dependent on piezo and/or on heartbeat, we analyzed endogenous Bnl expression using the bnl:GFP ${ }^{\text {endo }}$ knock-in allele ${ }^{35}$. When the heart was arrested or piezo knocked down in cardiac cells, more bnl:GFP ${ }^{\text {endo }}$ was observed in cardiac cells (Fig. 2a-e), indicating that piezo and heartbeat control $\mathrm{Bnl}$ protein levels in cardiac tube. We then established that Piezo and heartbeat repressed $b n l$ transcription in cardiac cells, since $b n l$ transcription, quantified by in situ hybridization, was higher when either piezo was knocked down or the heart arrested (Fig. 2f-j).

To investigate the functional link between bnl and piezo in cardiac cells and lymph gland hematopoiesis, we monitored blood cell differentiation using crystal cell differentiation as a readout, and asked whether we could rescue the decreased crystal cell numbers due to piezo knockdown by knocking down $b n l$ in cardiac cells. While the expression of $b n l$-RNAi in cardiac cells increased crystal cell numbers, in agreement with the previous report ${ }^{14}$, and piezo-RNAi reduced crystal cell numbers, simultaneous expression of bnl-RNAi and piezoRNAi restored wild type crystal cell numbers (Fig. 2k-1, n). Altogether, these data indicate that Piezo functions by repressing $b n l$ expression. Furthermore, since heartbeat activates Piezo which represses Bnl expression, we wondered whether Bnl reduction in cardiac cells can 
compensate for heart arrest. Crystal cell differentiation returned to normal when $b n l-R N A i$ was expressed in a context where the heart was blocked (Hand $\Delta>$ bnl-RNAi $>$ Ork $1 \Delta \mathrm{C}$, Fig. 2m, o). In conclusion, both heartbeat and Piezo control lymph gland hematopoiesis by repressing $b n l$ expression in cardiac cells. All these data lead us to propose that heartbeat activates the mechanosensitive channel Piezo in aorta cells, which in turn represses bnl transcription and therefore regulates lymph gland homeostasis.

\section{Notch acts downstream of Piezo to repress bnl expression}

To further decipher the molecular machinery downstream of Piezo, we first analyzed $\mathrm{Ca}^{2+}$

152 levels, since Piezo triggers upregulation of cytosolic $\mathrm{Ca}^{2+} 36-39$. Using the $\mathrm{Ca}^{2+}$ sensor 153 GCaMP $^{40}$ we observed that knocking down piezo in cardiac cells (Fig. 3a-c) or blocking 154 heartbeat (Supplementary Fig. 3a-c) led to decreased fluorescence compared to control, reflecting reduced $\mathrm{Ca}^{2+}$ levels. Thus heartbeat, via blood flow, activates Piezo which in turn regulates cytosolic $\mathrm{Ca}^{2+}$ levels in cardiac cells.

157 We subsequently focused on Notch $(\mathrm{N})$ signaling since Piezo, by modulating $\mathrm{Ca}^{2+}$ levels, 158 regulates $\mathrm{N}$ signaling in several cell types such as mouse endothelial cells ${ }^{41}$, zebrafish cardiac 159 valve cells ${ }^{42}$ and Drosophila gut cells ${ }^{30}$. In Drosophila bnl transcription is repressed by N 160 signaling in the embryonic tracheal system $^{43}$. We therefore hypothesized that in cardiac cells Piezo could activate $\mathrm{N}$ signaling, which in turn would repress $b n l$ expression. We therefore 162 investigated the role of $\mathrm{N}$ signaling in cardiac cells. The Notch Responsive Element 163 (Notch[NRE]-GFP) ${ }^{44}$ is a N signaling responsive enhancer. It is expressed in cardiac cells 164 (Fig. 3d-d') dependent on $\mathrm{N}$ signaling, since it is reduced when a dominant negative form of 165 $\mathrm{N}\left(\mathrm{N}^{\mathrm{xho}}\right)$ is expressed in cardiac cells (Supplementary Fig. 3e-e', g). These data indicate that the $\mathrm{N}$ pathway is activated in cardiac cells. To strengthen this conclusion, we analyzed the 167 expression of NRE-GFP, a synthetic reporter for $\mathrm{N}$ pathway activation, and of $\mathrm{E}(\mathrm{spl}) \mathrm{mbeta-}$ 168 HLH-GFP, which reports on canonical N pathway activation ${ }^{45}$. For both reporters, GFP was 
expressed in cardiac cells, confirming that the canonical $\mathrm{N}$ pathway is activated in cardiac cells (Supplementary Fig. 3i-j'). When piezo was knocked down in cardiac cells (Fig. 3e-f) or when heartbeat was arrested (Supplementary Fig. 3f-f', h), Notch[NRE]-GFP expression was reduced establishing that heartbeat and piezo activate $\mathrm{N}$ signaling. Furthermore we showed that $\mathrm{N}$ signaling is dependent on $\mathrm{Ca}^{2+}$ levels, since Notch[NRE]-GFP expression was reduced when $\mathrm{Ca}^{2+}$ levels were decreased in cardiac cells. This was achieved using either CamKII knockdown (CamKII-RNAi) or with the inhibition of ER-mediated $\mathrm{Ca}^{2+}$ release in the cytosol by knocking down IP3R (IP3R-RNAi) ${ }^{46}$ (Fig. 3g-i). Collectively, these data indicate that Piezo through the control of intracellular $\mathrm{Ca}^{2+}$ levels activates $\mathrm{N}$ signaling. We then investigated the N/Bnl relationship. Bnl levels, as visualized with bnl:GFP ${ }^{\text {endo }}$, were higher in cardiac tube when the $\mathrm{N}$ pathway was inhibited $\left(\mathrm{N}^{\mathrm{Xho}}\right.$, Fig. $\left.3 \mathrm{j}-1\right)$ or when $N-R N A i$ was expressed in cardiac cells (Supplementary Fig. 3k-m). This indicates that $\mathrm{N}$ signaling represses $b n l$ expression in cardiac cells.

We asked whether $\mathrm{N}$ signaling in cardiac cells is required for lymph gland homeostasis. We monitored blood cell differentiation using crystal cell differentiation as a readout. When $\mathrm{N}$ signaling was inhibited by expressing $\left(\mathrm{N}^{\mathrm{xho}}\right)$ with either of the two heart drivers (Fig. 3m, o and Supplementary Fig. 3n-o, q), crystal cell numbers were lower than in the control. Reduced crystal cell differentiation was also seen upon expression of $N-R N A i$ in cardiac cells (Supplementary Fig. 3r-t) or of a dominant negative form of mastermind $\left(\mathrm{mam}^{D N}\right)$. Mam is the coactivator of NICD to regulate $\mathrm{N}$ target gene expression ${ }^{45}$ (Supplementary Fig. 3p-q). In conclusion, the $\mathrm{N}$ signaling pathway is activated in cardiac cells and regulates lymph gland hematopoiesis non-cell autonomously via Bnl. Piezo activation and $\mathrm{N}$ loss-of-function have opposite effects on crystal cell numbers. Simultaneous expression of a constitutively active form of Piezo (mpiezo1-TriM) and $\mathrm{N}^{\mathrm{Xho}}$ ( $\mathrm{N}$ inhibition) led to increased crystal cell numbers compared to inactivation of $\mathrm{N}$ signaling alone (Fig. 3n-o), establishing that Piezo and $\mathrm{N}$ are 
functionally linked and that Piezo in part functions through N signaling. Finally, knockdown of $b n l$ in cardiac cells compensates for the crystal cell differentiation defect due to $\mathrm{N}$ signaling inhibition, indicating that $\mathrm{N}$ pathway regulates crystal cell differentiation via the repression of $b n l$ transcription in cardiac cells (Fig. 3p-r).

In conclusion, our data are consistent with the hypothesis that in cardiac cells Piezo activates canonical $\mathrm{N}$ signaling, which subsequently represses $b n l$ expression, which in turn regulates lymph gland hematopoiesis (Fig. 3s).

\section{A physiological chronotropic effect controls lymph gland hematopoiesis}

We finally wondered what could be the physiological relevance of a control of lymph gland hematopoiesis by heartbeat. When measuring heartbeat rate, we discovered that it increases slightly during larval development (Fig. 4a and movies 7-8). We then looked at Bnl expression and observed more abundant bnl:GFP ${ }^{\text {endo }}$ in the cardiac tube of late L2 larvae compared to mid L3 larvae (Fig. 4b-d), indicating that Bnl levels in the cardiac tube decrease between L2 and L3 larval stages. While there were no crystal cells in late L2 lymph glands, many were found in mid L3 larvae (Fig. 4e-g), confirming published data indicating that blood cell differentiation occurs in L3 larvae, mature blood cells being seldom found in L2 larvae $e^{13}$. Finally, we determined whether temporal differentiation of crystal cells is dependent on heartbeat rate and/or Piezo activation. We showed that premature blood cell differentiation occurs in late L2 larvae when heartbeat rate was increased (Ork1-RNAi) and when Piezo was constitutively activated (mPiezo1-TriM) from L1 to L2 larval stages (Fig. 4h-j). All these data strongly suggest that the temporal control, between L2 and L3 larval stages, of lymph gland blood cell differentiation may be achieved through physiological modulation of the heartbeat rate.

In conclusion, cardiac cells, previously established to act as vascular niche cells, may directly sense mechanical forces provided by blood flow and adjust lymph gland 
hematopoiesis accordingly. This mechanosensing is mediated by Piezo activation that transduces the stimuli into cellular responses in vascular niche cells. Our findings show that Piezo, activated by blood flow, promotes blood progenitor maintenance via $\mathrm{N}$ activation in vascular niche cells, which in turn represses expression of the FGF ligand Bnl. Whether N signaling represses $b n l$ transcription in cardiac cells via $E(s p l)$ genes has to be confirmed. Bnl secreted by vascular niche cells non-cell-autonomously activates the FGF pathway in hematopoietic progenitors for their maintenance. These data demonstrate a direct link between the blood flow rate and the maintenance of hematopoietic progenitors, and provide a regulatory network involving mechanosensitive channels in hematopoietic vascular niche cells to promote blood cell progenitor maintenance. Given the striking parallels between Drosophila lymph gland and mammalian bone marrow hematopoiesis, there is promise that our newly identified regulations are conserved and that mechanosensing of blood flow by endothelial cells, a major cell type of the bone marrow vascular niche in mammals, may also contribute to HSPC maintenance. Modification of blood pressure, such as during physical activity, could therefore be a powerful way to regulate hematopoiesis.

\section{Materials and Methods.}

Fly strains. $w^{1118}$ (wild type, WT), UAS-mcD8GFP ${ }^{19}$, hand $\Delta$-gal4 ${ }^{14}$, NP1029-gal4 ${ }^{47}$, BcGFP $^{48}$, bnl:GFP ${ }^{\text {endo }} 35$, HandCGFP ${ }^{49}$, UAS-Ork1 $\triangle \mathrm{C}$ and UAS-Ork1-RNAi ${ }^{17}$, UAS-PiezoRNAi1 (line 1), UAS-mPiezo1-2336-Myc and UAS-mPiezo1-TriM ${ }^{31}$, Mhc-RNAi1 (line 1) (F.Schnörrer), Notch[NRE]-GFP and Notch-RNAi ${ }^{44}$, NRE-GFP and UAS-Mam ${ }^{\text {DN }}{ }^{45}$, and $\mathrm{N}^{\text {xho }}$ (S.Bray). Other strains were provided by Bloomington (BL) and Vienna (VDRC) Drosophila stock centers: UAS-GCaMP3 (BL32116), UAS-Dicer (BL24650), 76E11-gal4 (BL39933), tub-gal80 ${ }^{\text {ts }}$ (BL7019), UAS-piezo-RNAi2 (line 2) (VDRC v25780), Mhc-RNAi2 (line 2) (BL26299), piezo-gal4 (KI) (BL78335), piezo-gal4 (enhancer) (BL59266), piezo 
(KO) (BL58770), UAS-bnl-RNAi (VDRC GD5730), UAS-CaMKII (BL29401), UAS-IP3R-

RNAi (BL25937) and E(spl)mbeta-HLH-GFP (BL65294). Crosses and subsequent raising of larvae until late L1/early L2 stage were performed at $22^{\circ} \mathrm{C}$, before shifting larvae to $25^{\circ} \mathrm{C}$ until their dissection at L3 stage. For RNAi treatments the same procedure was followed except that larvae were shifted to $29^{\circ} \mathrm{C}$ until their dissection. UAS-Dicer was introduced and at least two independent RNAi lines per gene were tested. Controls correspond to Gal4 drivers with UAS-Dicer crossed with $w^{1118}$. For gal $80^{\text {ts }}$ experiments, crosses were initially maintained at $18^{\circ} \mathrm{C}$ (permissive temperature) for 3 days after egg laying, and then shifted to $29^{\circ} \mathrm{C}$ until dissection.

Antibodies. Primary antibodies were mouse anti-Hnt (1/100, Hybridoma Bank), mouse antiCol (1/40) ${ }^{19}$, mouse anti-P1 (1/30, I. Ando, Institute of Genetics, Biological Research Center of the Hungarian Academy of Science, Szeged, Hungary), mouse anti-Antp (1/100, Hybridoma Bank), chicken anti-GFP (1/500, Abcam). Secondary antibodies were Alexa Fluor-488 and -555 conjugated antibodies (1/1000, Molecular Probes) and goat anti-Chicken Alexa Fluor-488 (1/800, Molecular Probes). Nuclei were labeled with TOPRO3 (Thermo Fisher Scientific). For detecting bnl:GFP ${ }^{\text {endo }}$ and Notch[NRE]-GFP, GFP immunostainings were performed.

Heart rate measurements and movies. Larvae expressed HandC-GFP with GFP labelling of cardioblasts and pericardial cells. Larvae were anaesthetized with FlyNap (Carolina Biological, Burlington, NC, USA). $5 \mu$ of FlyNap put on a piece of cotton in a closed chamber and larvae put in chamber for 160 seconds. Anaesthetized larvae deposited on a glass slide for recording with a binocular macroscope (SMZ18, Nikon). Anaesthetized larvae kept at $25^{\circ} \mathrm{C}$ for 10 minutes before recording. For each individual, 20 second-recordings performed, and this repeated twice with 10 minutes of rest in the dark. To measure heart contraction, movies were converted into a kymograph using Fiji software. For each larva, number of heartbeat 
contractions during 20 seconds corresponds to average of three measurements. At least 10 larvae per genotype scored and experiments reproduced at least three times. All recorded larvae survived and gave rise to an adult. Statistical analyses $t$-test (Mann-Whitney nonparametric test) performed using GraphPad Prism 5 software.

Quantifications. In all experiments, optimized confocal sections performed on Leica SP8 microscope for 3D reconstructions, and since number of lymph gland differentiated blood cells fluctuates depending on the larval stage, and to limit discrepancies, all genotypes always analyzed in parallel. For all quantifications, statistical analyses $t$-test (Mann-Whitney nonparametric test) performed using GraphPad Prism 5 software.

\section{Blood cell and progenitor quantification.}

Crystal cells visualized by BcGFP or immunostaining with antibodies against Hnt. Plasmatocytes and core progenitors labelled by $\mathrm{P} 1$ and Col immunostaining, respectively. Nuclei labelled by TOPRO3. Number of crystal cells and volume (in $\mu \mathrm{m}^{3}$ ) of each anterior lymph gland lobe measured using Fiji software and 3DSuite plugin ${ }^{50}$. Crystal cell index corresponds to [(number of crystal cells)/(primary lobe volume)] x100. Plasmatocyte or progenitor index corresponds to (plasmatocyte or progenitor volume/anterior lobe volume) $\mathrm{x}$ 10. At least 15 anterior lobes scored per genotype, and experiments reproduced at least three times.

\section{Quantification of UAS-GCaMP3 intensity.}

Volume of cardiac tube localized in between two lymph gland anterior lobes measured. For GCaMP3, mean intensity of cytoplasmic GFP quantified using Fiji software and the 3DSuite plugin ${ }^{50}$. Mean intensity of GCaMP3 corresponds to sum intensity of GCaMP3 per cardiac tube volume. At least 20 cardiac tubes scored per genotype, and experiments reproduced at least three times.

\section{Quantification of bnl::GFP and Notch[NRE]-GFP dots, and $b n l$ expression.}


A ROI including cardiac tube in between the anterior lymph gland lobes was selected. To normalize with respect to background in tissue preparations, a ROI in the lymph gland cortical zone was selected and used as background level. Volume of ROI and number of Bnl::GFP or Notch[NRE]-GFP granules per ROI was quantified using Fiji software and DiAna plugin ${ }^{51}$. The sum of GFP granules per volume was calculated. Notch[NRE]-GFP and bnl::GFP levels correspond to GFP granules measured in the cardiac tube minus the sum of granules measured in the cortical zone. At least 10 cardiac tubes scored per genotype. For $b n l$ expression levels, volume of cardiac tube and $b n l$ fluorescence quantified using Fiji software and 3DSuite plugin $^{50} . b n l$ mean intensity corresponds to $b n l$ fluorescence/volume. At least 6 cardiac tubes scored per genotype. All experiments reproduced at least three times.

Data availability. The authors declare that the data supporting the findings of this study are available within the article and its Extended Data Figs.

\section{References}

1. Acar, M. et al. Deep imaging of bone marrow shows non-dividing stem cells are mainly perisinusoidal. Nature 526, 126-30 (2015).

2. Baryawno, N. et al. A Cellular Taxonomy of the Bone Marrow Stroma in Homeostasis and Leukemia. Cell 177, 1915-1932 e16 (2019).

3. Crane, G.M., Jeffery, E. \& Morrison, S.J. Adult haematopoietic stem cell niches. Nat Rev Immunol 17, 573-590 (2017).

4. Pinho, S. \& Frenette, P.S. Haematopoietic stem cell activity and interactions with the niche. Nat Rev Mol Cell Biol 20, 303-320 (2019).

5. Ramasamy, S.K. et al. Regulation of Hematopoiesis and Osteogenesis by Blood VesselDerived Signals. Annu Rev Cell Dev Biol 32, 649-675 (2016).

6. Tikhonova, A.N. et al. The bone marrow microenvironment at single-cell resolution. Nature 569, 222-228 (2019).

7. Comazzetto, S., Shen, B. \& Morrison, S.J. Niches that regulate stem cells and hematopoiesis in adult bone marrow. Dev Cell (2021).

8. Kunisaki, Y. et al. Arteriolar niches maintain haematopoietic stem cell quiescence. Nature 502, 637-43 (2013).

9. Horton, P.D., Dumbali, S. \& Wenzel, P.L. Mechanoregulation in hematopoiesis and hematologic disorders. Curr Stem Cell Rep 6, 86-95 (2020).

10. Shen, B. et al. A mechanosensitive peri-arteriolar niche for osteogenesis and lymphopoiesis. Nature 591, 438-444 (2021).

11. Letourneau, M. et al. Drosophila hematopoiesis under normal conditions and in response to immune stress. FEBS Lett 590, 4034-4051 (2016). 
12. Morin-Poulard, I., Tian, Y., Vanzo, N. \& Crozatier, M. Drosophila as a Model to Study Cellular Communication Between the Hematopoietic Niche and Blood Progenitors Under Homeostatic Conditions and in Response to an Immune Stress. Front Immunol 12, 719349 (2021).

13. Banerjee, U., Girard, J.R., Goins, L.M. \& Spratford, C.M. Drosophila as a Genetic Model for Hematopoiesis. Genetics 211, 367-417 (2019).

14. Destalminil-Letourneau, M., Morin-Poulard, I., Tian, Y., Vanzo, N. \& Crozatier, M. The vascular niche controls Drosophila hematopoiesis via fibroblast growth factor signaling. Elife 10(2021).

15. Rotstein, B. \& Paululat, A. On the Morphology of the Drosophila Heart. J Cardiovasc Dev Dis 3(2016).

16. Choma, M.A., Suter, M.J., Vakoc, B.J., Bouma, B.E. \& Tearney, G.J. Physiological homology between Drosophila melanogaster and vertebrate cardiovascular systems. Dis Model Mech 4, 411-20 (2011).

17. Lalevee, N., Monier, B., Senatore, S., Perrin, L. \& Semeriva, M. Control of cardiac rhythm by ORK1, a Drosophila two-pore domain potassium channel. Curr Biol 16, 1502-8 (2006).

18. Perrin, L., Monier, B., Ponzielli, R., Astier, M. \& Semeriva, M. Drosophila cardiac tube organogenesis requires multiple phases of Hox activity. Dev Biol 272, 419-31 (2004).

19. Krzemien, J. et al. Control of blood cell homeostasis in Drosophila larvae by the posterior signalling centre. Nature 446, 325-8 (2007).

20. Oyallon, J. et al. Two Independent Functions of Collier/Early B Cell Factor in the Control of Drosophila Blood Cell Homeostasis. PLoS One 11, e0148978 (2016).

21. Pennetier, D. et al. Size control of the Drosophila hematopoietic niche by bone morphogenetic protein signaling reveals parallels with mammals. Proc Natl Acad Sci U S A 109, 3389-94 (2012).

22. Mandal, L., Martinez-Agosto, J.A., Evans, C.J., Hartenstein, V. \& Banerjee, U. A Hedgehog- and Antennapedia-dependent niche maintains Drosophila haematopoietic precursors. Nature 446, 320-4 (2007).

23. Morin-Poulard, I. et al. Vascular control of the Drosophila haematopoietic microenvironment by Slit/Robo signalling. Nat Commun 7, 11634 (2016).

24. Tokusumi, Y., Tokusumi, T., Stoller-Conrad, J. \& Schulz, R.A. Serpent, suppressor of hairless and U-shaped are crucial regulators of hedgehog niche expression and prohemocyte maintenance during Drosophila larval hematopoiesis. Development 137, 3561-8 (2010).

25. Baldeosingh, R., Gao, H., Wu, X. \& Fossett, N. Hedgehog signaling from the Posterior Signaling Center maintains U-shaped expression and a prohemocyte population in Drosophila. Dev Biol (2018).

26. Benmimoun, B., Polesello, C., Haenlin, M. \& Waltzer, L. The EBF transcription factor Collier directly promotes Drosophila blood cell progenitor maintenance independently of the niche. Proc Natl Acad Sci U S A 112, 9052-7 (2015).

27. Coste, B. et al. Piezo proteins are pore-forming subunits of mechanically activated channels. Nature 483, 176-81 (2012).

28. Volkers, L., Mechioukhi, Y. \& Coste, B. Piezo channels: from structure to function. Pflugers Arch 467, 95-9 (2015).

29. Kim, S.E., Coste, B., Chadha, A., Cook, B. \& Patapoutian, A. The role of Drosophila Piezo in mechanical nociception. Nature 483, 209-12 (2012).

30. He, L., Si, G., Huang, J., Samuel, A.D.T. \& Perrimon, N. Mechanical regulation of stem-cell differentiation by the stretch-activated Piezo channel. Nature 555, 103-106 (2018).

31. Song, Y. et al. The Mechanosensitive Ion Channel Piezo Inhibits Axon Regeneration. Neuron 102, 373-389 e6 (2019).

32. Tokusumi, Y., Tokusumi, T. \& Schulz, R.A. The nociception genes painless and Piezo are required for the cellular immune response of Drosophila larvae to wasp parasitization. Biochem Biophys Res Commun 486, 893-897 (2017). 
33. Suslak, T.J. et al. Piezo Is Essential for Amiloride-Sensitive Stretch-Activated Mechanotransduction in Larval Drosophila Dorsal Bipolar Dendritic Sensory Neurons. PLoS One 10, e0130969 (2015).

34. McGuire, S.E., Mao, Z. \& Davis, R.L. Spatiotemporal gene expression targeting with the TARGET and gene-switch systems in Drosophila. Sci STKE 2004, pl6 (2004).

35. Du, L., Sohr, A., Yan, G. \& Roy, S. Feedback regulation of cytoneme-mediated transport shapes a tissue-specific FGF morphogen gradient. Elife 7(2018).

36. Cinar, E. et al. Piezo1 regulates mechanotransductive release of ATP from human RBCs. Proc Natl Acad Sci U S A 112, 11783-8 (2015).

37. Li, J. et al. Piezo1 integration of vascular architecture with physiological force. Nature 515, 279-282 (2014).

38. Gudipaty, S.A. et al. Mechanical stretch triggers rapid epithelial cell division through Piezo1. Nature 543, 118-121 (2017).

39. Pathak, M.M. et al. Stretch-activated ion channel Piezo1 directs lineage choice in human neural stem cells. Proc Natl Acad Sci U S A 111, 16148-53 (2014).

40. Nakai, J., Ohkura, M. \& Imoto, K. A high signal-to-noise $\mathrm{Ca}(2+)$ probe composed of a single green fluorescent protein. Nat Biotechnol 19, 137-41 (2001).

41. Caolo, V. et al. Shear stress activates ADAM10 sheddase to regulate Notch1 via the Piezo1 force sensor in endothelial cells. Elife 9(2020).

42. Duchemin, A.L., Vignes, H. \& Vermot, J. Mechanically activated piezo channels modulate outflow tract valve development through the Yap1 and KIf2-Notch signaling axis. Elife 8(2019).

43. Ikeya, T. \& Hayashi, S. Interplay of Notch and FGF signaling restricts cell fate and MAPK activation in the Drosophila trachea. Development 126, 4455-63 (1999).

44. Simon, R., Aparicio, R., Housden, B.E., Bray, S. \& Busturia, A. Drosophila p53 controls Notch expression and balances apoptosis and proliferation. Apoptosis 19, 1430-43 (2014).

45. Zacharioudaki, E. \& Bray, S.J. Tools and methods for studying Notch signaling in Drosophila melanogaster. Methods 68, 173-82 (2014).

46. Shim, J. et al. Olfactory control of blood progenitor maintenance. Cell 155, 1141-53 (2013).

47. Monier, B., Astier, M., Semeriva, M. \& Perrin, L. Steroid-dependent modification of Hox function drives myocyte reprogramming in the Drosophila heart. Development 132, 5283-93 (2005).

48. Tokusumi, T., Shoue, D.A., Tokusumi, Y., Stoller, J.R. \& Schulz, R.A. New hemocyte-specific enhancer-reporter transgenes for the analysis of hematopoiesis in Drosophila. Genesis 47, 771-4 (2009).

49. Sellin, J., Albrecht, S., Kolsch, V. \& Paululat, A. Dynamics of heart differentiation, visualized utilizing heart enhancer elements of the Drosophila melanogaster bHLH transcription factor Hand. Gene Expr Patterns 6, 360-75 (2006).

50. Ollion, J., Cochennec, J., Loll, F., Escude, C. \& Boudier, T. TANGO: a generic tool for highthroughput 3D image analysis for studying nuclear organization. Bioinformatics 29, 1840-1 (2013).

51. Gilles, J.F., Dos Santos, M., Boudier, T., Bolte, S. \& Heck, N. DiAna, an ImageJ tool for objectbased 3D co-localization and distance analysis. Methods 115, 55-64 (2017).

\section{Acknowledgements}

We thank H. Bouhkatmi, S. Bray, F. Schnorrer, Y. N. Jan, S. Roy, Bloomington and Vienna

Stock Center and the TRiP at Harvard Medical School for fly strains; I. Ando, A. Moore and 
T. Trenczek for antibodies,. M. Haenlin, G. Lebreton, M. Meister, B. Monier, C. Monod, A.

430 Vincent and X. Wang for critical reading of the manuscript. We are grateful to B. Ronsin and

431 S. Bosch for assistance with confocal microscopy (Platform TRI), J. Favier, V. Nicolas and A.

432 Destenable for fly culture and B. Monier and X. Wang for their advice during the course of Y.

433 Tian's thesis. Research in the authors' laboratory is supported by the CNRS, University

434 Toulouse III, FRM (Fondation pour la Recherche Médicale), La Ligue Contre le Cancer 31,

435 La Société Française d'Hématologie (SFH), the China Scholarship Council and the CNRS

436 «Groupement de recherche $3740 »$.

\section{Author contributions}

438 Conceived and designed the experiments: Y.T. and M.C. performed the experiments: Y.T.

439 and I.M.P.. Analyzed the data: Y.T., I.M.P., N.V. and M.C.. Contributed 440 reagents/materials/analysis tools: Y.T. and I.M.P.. Wrote the paper :Y.T. and M.C..

441 Competing interests: The authors declare no conflict of interest.

Correspondence and requests for materials should be addressed to $\mathrm{M}$. Crozatier.

443

Figure 1: Heartbeat and Piezo expressed in cardiac cells control lymph gland homeostasis.

(a) The lymph gland is composed of progenitors (red) and core progenitors (hatched red) in a medullary zone (MZ), and a cortical zone which contains differentiated hemocytes (CZ, dots). The two niches, the PSC (pink) and the cardiac tube (orange) regulate different subsets of MZ progenitors (black arrows). (b) Kymograph of heartbeat in control and when Ork1 $\Delta C$, Mhc$R N A i$ and $O r k 1-R N A i$ are expressed with cardiac cell drivers. (c-d) Heart contraction numbers per 20s. (e-h) Crystal cell differentiation (Hnt, green) when the heart is blocked (f-g) or accelerated (h). (i) Crystal cell index. (j-k, m) Col (red,*) labels core progenitors and the PSC (arrow). (1, o) Quantification. (n) mcd8-GFP (green) under piezo-Gal4 is expressed in cardiac cells (arrowhead). (p-q) Fewer crystal cells (Hnt, green) in a piezo null mutant (p) or when 
piezo is knocked down in cardiac cells (q). ( $\mathrm{r}-\mathrm{s})$ Crystal cell index. ( $\mathrm{t}-\mathrm{u})$ Active form of piezo (mPiezo1-TriM) with arrested heart $(\operatorname{Ork1} \Delta C)$ restores wild type crystal cell numbers (u). (v)

Crystal cell index. Statistical analysis $t$-test (Mann-Whitney nonparametric test) performed using GraphPad Prism 5 software. Error bars represent SEM and $* \mathrm{P}<0.1$; ** $\mathrm{P}<0.01$; ***P $<0.001 ; * * * * \mathrm{P}<0.0001$. ns (not significant). Nuclei labelled with Topro (blue) and scale bars $=20 \mu \mathrm{m}$.

Figure 2: Heartbeat and Piezo control expression of FGF ligand Branchless (Bnl) in cardiac cells and in turn Bnl regulates lymph gland hematopoiesis.

(a-c') Enlarged view of larval cardiac tube expressing bnl:GFP $P^{\text {endo }}$ (green) and Col (red) in core progenitors (a-c) and bnl:GFP endo (white) in (a'-c'). Dotted lines indicate cardiac tube contour. bnl:GFP endo expression is higher when heart is blocked (b-c'). (d-e) Quantification. (f-h') Enlarged view of cardiac tube when piezo-RNAi (g-g') or Ork1 $\Delta \mathrm{C}$ (h-h') expressed in cardiac cells; $b n l$ in red (f-h) and white (f'-h'). $b n l$ expression in cardiac cells increases in both conditions. (i-j) Quantification. (k-m) Crystal cell (Hnt, green) differentiation increases when bnl knocked down in cardiac cells $(\mathrm{k})$ and restored to wild type when piezo and bnl simultaneously knocked down in cardiac cells (1). Crystal cell differentiation rescued when $b n l$ knocked down in cardiac cells simultaneously with heart arrest (m). (n-o) Crystal cell index.

Figure 3: Piezo regulates $\mathrm{Ca}^{2+}$ levels and activates $\mathrm{N}$ signaling, which controls blood cell differentiation by repressing $b n l$ transcription in cardiac cells. (a-b) GCaMP3 $\mathrm{Ca}^{2+}$ sensor (green) decreases when piezo knocked down in cardiac cells. (c) Quantification. (d-e', g-h') Enlarged view of larval cardiac tube expressing Notch[NRE]-GFP (green) and $\mathrm{Col}(\mathrm{red})$ in core progenitors (d-e, g-h) and Notch[NRE]-GFP (white) in (d'-e', g'-h'). Dotted lines indicate cardiac tube contour. Notch[NRE]-GFP expression decreased when piezo knocked down (e-e') and when $\mathrm{Ca}^{2+}$ levels decreased (g-h') in cardiac cells. (f, i) Quantification. (j-k') bnl:GFP endo expression increased when $\mathrm{N}$ signaling inhibited. (1) 
Quantification. (m-n, p-q) Crystal cell (Hnt, green) differentiation reduced when N signaling inhibited (m), and rescued when simultaneously $\mathrm{N}$ signaling is inhibited and Piezo is constitutively activated (n). Crystal cell differentiation defect observed when $\mathrm{N}$ is inhibited $\left(N^{x h o}\right)$, is rescued when simultaneously $\mathrm{N}$ signaling is inhibited and $b n l$ is knocked down (q). (o, r) Crystal cell index. (s) Representation of third instar larval lymph gland anterior lobe. Blood flow resulting from heartbeat activates Piezo in cardiac/vascular cells leading to $\mathrm{Ca}^{2+}$ increase which activates $\mathrm{N}$ signaling, which in turn represses $b n l$ expression in vascular cells. Bnl normally produced by vascular cells activates FGF pathway in progenitors, where it is required for their maintenance at the expense of their differentiation.

Figure 4: Physiological modulation of heartbeat rate during larval development regulates hematopoiesis.

(a) Heart contraction number per 20 seconds in late L2 and mid L3 larvae. (b-c') Enlarged view of cardiac tube in larvae expressing bnl:GFP endo (green) and Col (red) in core progenitors (b, c) and bnl:GFP endo (white) in (b', c') in late L2 (b, b') and mid L3 larvae (c, c'). Dotted lines indicate cardiac tube contour. bnl:GFP endo decreases in mid L3 compared to late L2 larvae. (d) Quantification. (e-f, h-i) Hnt (green) labels crystal cells. While crystal cells are seldom found in late L2 (e), they massively differentiate in mid L3 larvae (f). Premature crystal cell differentiation is observed in late L2 larvae when heartbeat accelerated (h) or when a constitutively activated form of Piezo expressed in cardiac cells (i). (g, j) Crystal cell index.

\section{Supplementary Figures}

Figure S1: Heartbeat regulates lymph gland homeostasis.

(a) Cardiac cells express mcd8-GFP (NP1029>mcD8-GFP, green). Transversal section shown. No difference in cardiac tube morphology observed when heartbeat arrested (ORK1 $\triangle \mathrm{C}$ or Mhc-RNAi). (b) Heart contraction number per 20 seconds when heart arrested 
Plasmatocyte index. (f, g) Hnt (green) labels crystal cells. Compared to the control (f) fewer crystal cells are observed when heart arrested (Hand $\Delta>\operatorname{Ork1} 1 \Delta C$, g). (h) Crystal cell index. (iwhen heart arrested (NP1029>Mhc-RNAi, j). (k) Crystal cell index. (1-m) Col (red) is expressed at high levels in the PSC (arrow) and at lower levels in core progenitors (*). Compared to the control (1) higher levels of $\mathrm{Col}$ are observed in core progenitors when heart arrested (Hand $>$ Orkl $\triangle C$, m). (n) Core progenitor index. (o-p) Enlargement of PSC cells labelled by Antp (red) antibody. No difference in PSC cell numbers is observed when heart arrested (p) compared to the control (o). (q) Quantification of PSC cell number.

\section{Figure S2: Piezo expressed in anterior aorta cells controls lymph gland hematopoiesis.}

(a-b) piezo(enhancer) $>m c D 8-G F P$ and (c) piezo-gal4(KI)>mcd8-GFP. Col (red) labels PSC

(white arrow), lymph gland posterior lobes (red arrows) and pericardial cells (PC). In L3 larvae, piezo is expressed in cardiac cells (white arrowhead). (b) Enlargement of lymph gland cortical zone expressing piezo-gal4>mcd8-GFP (green), piezo is expressed in crystal cells labelled by Hnt (red). (d) In piezo null mutant, crystal cell (Hnt, green) differentiation is decreased. (e) Crystal cell index. (f-g) Col (red) is expressed at high levels in the PSC (arrow) and at lower levels in core progenitors $(*)$. Compared to the control (f), number of core progenitors increases when piezo knocked down in cardiac cells (g). (h) Core progenitor index. (i-j, m-n) BcGFP (green, i-j) or Hnt (green, m-n) labels crystal cells. Compared to the controls $(\mathrm{i}, \mathrm{m})$, fewer crystal cells are observed when piezo knocked down in cardiac cells $(\mathrm{j})$ or when a nonfunctional channel encoded by mPiezo1-2336-Myc expressed in cardiac cells 
larvae, 76E11 is expressed in anterior aorta cells (green). (q-r) Hnt (green) labels crystal cells.

532 Compared to the control (q), fewer crystal cells are observed when piezo knocked down (r) in cardiac cells using the 76E11-gal4 driver. (s) Crystal cell index. (t) Kymograph of heartbeat in control (hand $\Delta>$ ) and when piezo knocked down in cardiac cells. (u) Number of heart contractions per 20 seconds. No difference compared to the control is observed when piezo knocked down in cardiac cells.

\section{Figure S3: Heartbeat activates $\mathrm{N}$ signaling which regulates blood cell differentiation.}

(a-b) GCaMP3 $\mathrm{Ca}^{2+}$ sensor (green) expressed under NP1029 driver. GCaMP3 intensity decreases when heart arrested (b) compared to the control (a). (c) Quantification of GCaMP3 mean intensity. (d-f') Enlarged view of cardiac tube and a maximum projection of 10 confocal lymph gland sections of larvae expressing Notch[NRE]-GFP (green) and Col (red) that labels core progenitors (d-f) and Notch[NRE]-GFP (white) in (d'-f'). Dotted lines indicate cardiac tube contour. Compared to the control (d-d'), Notch[NRE]-GFP in cardiac cells decreases when $\mathrm{N}$ signaling inhibited in cardiac cells (e-e') or when and heart arrested (f-f'). (g-h) Quantification of Notch[NRE]-GFP levels in cardiac cells. (i-j') Enlarged view of cardiac tube of larvae expressing NRE-GFP (green, i) or E(spl)mbeta-GFP (green, j) and Col (red) that labels core progenitors. For $N R E-G F P$ and $E(s p l) m b e t a-G F P$, a maximum projection of 9 and 7 confocal lymph gland sections were used, respectively. (k-l') Enlarged view of cardiac tube and a maximum projection of 10 confocal lymph gland sections of larvae expressing bnl:GFP $P^{\text {endo }}$ (green) and Col (red) in core progenitors (k-l) and bnl:GFP endo (white) in (k'-l'). bnl:GFP $P^{\text {endo }}$ expression increases when $\mathrm{N}$ signaling inhibited in cardiac cells (1-1') compared to the control (k-k'). (m) Quantification in cardiac cells of bnl:GFP endo cytoplasmic granules relative to volume. (n-p, r-s) Hnt (green) labels crystal cells. Compared to controls (n, r), crystal cell differentiation decreases when the $\mathrm{N}$ pathway inhibited by expressing a dominant 
555 negative form of $\mathrm{N}\left(\mathrm{N}^{\mathrm{xho}}, \mathrm{o}\right)$, a dominant negative form of $\operatorname{mam}\left(\mathrm{mam}^{D N}, \mathrm{p}\right)$, or $N-R N A i(\mathrm{~s})$ in 556 cardiac cells. (q, t) Crystal cell index.

557 Movie 1: heartbeat in control NP1029; $m c D 8-G F P>$

558 Movie 2: heart is arrested in NP1029, $m c D 8-G F P>O r k 1 \triangle C$

559 Movie 3: heart is arrested in NP1029, $m c D 8-G F P>M h c-R N A i$

560 Movie 4: heartbeat in control Hand $\triangle$; HandC-GFP>

561 Movie 5: heartbeat is increased in Hand 4 ; HandC-GFP>Ork1-RNAI

562 Movie 6: heartbeat in Hand 4 ; HandC-GFP >piezo- RNAi

563 Movie 7: heartbeat in L2 larvae (Hand 4 ; HandC-GFP>)

564 Movie 8: heartbeat in L3 larvae (Hand 4 ; HandC-GFP>)

565 
Fig1

a
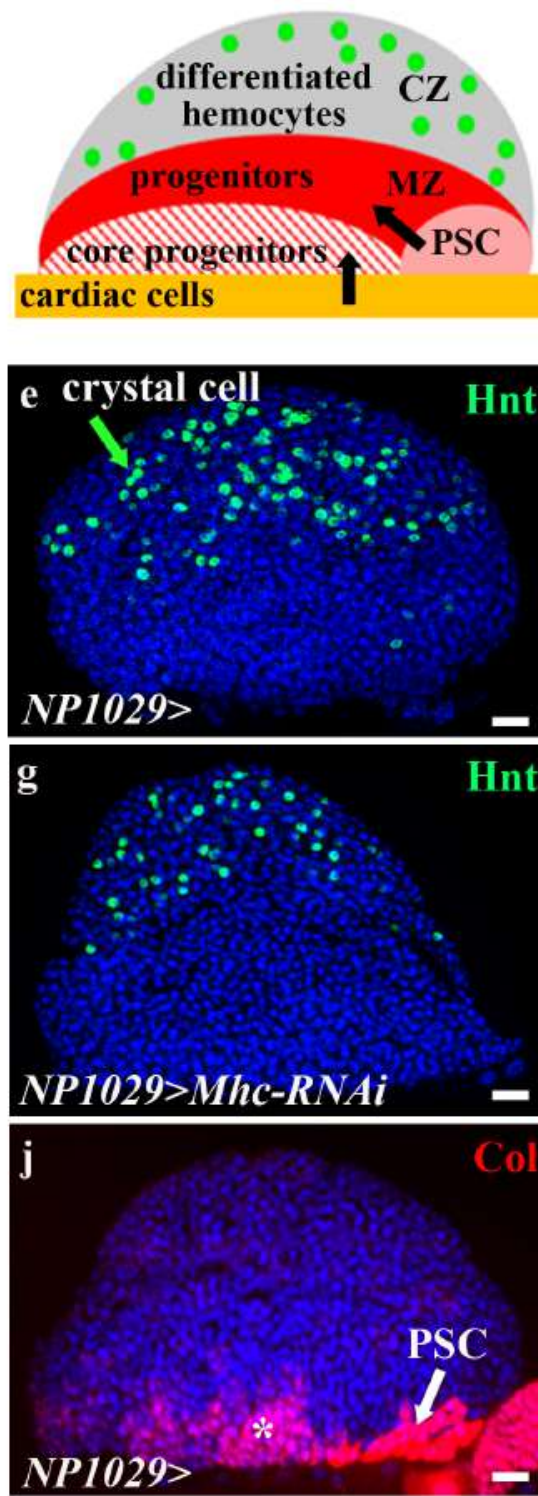

m

Hnt

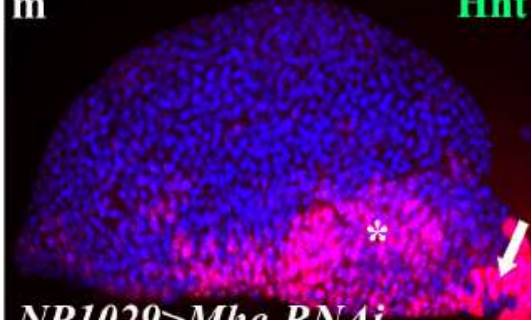

NP1029>Mhc-RNAi

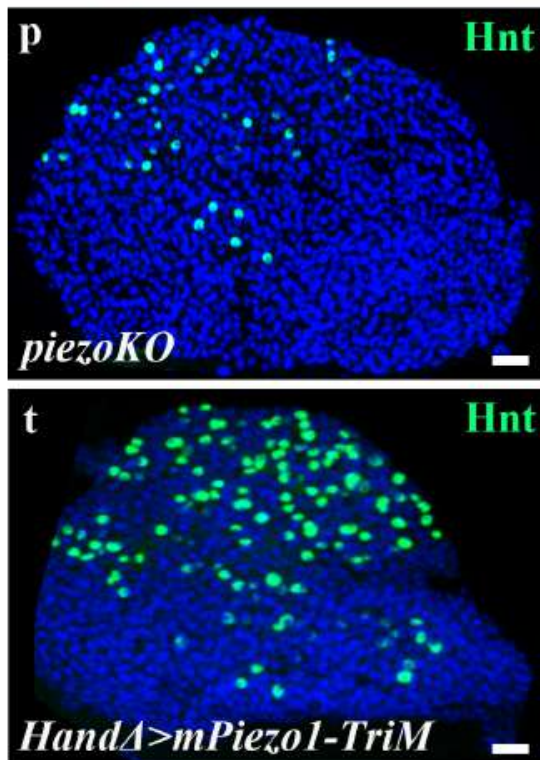

b

NP1029>

NP1029>

Ork14C

NP1029>

Mhc-RNA

Hand $\triangle>$

ORK-RNA

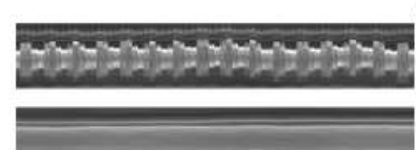

$A i=$

(1)
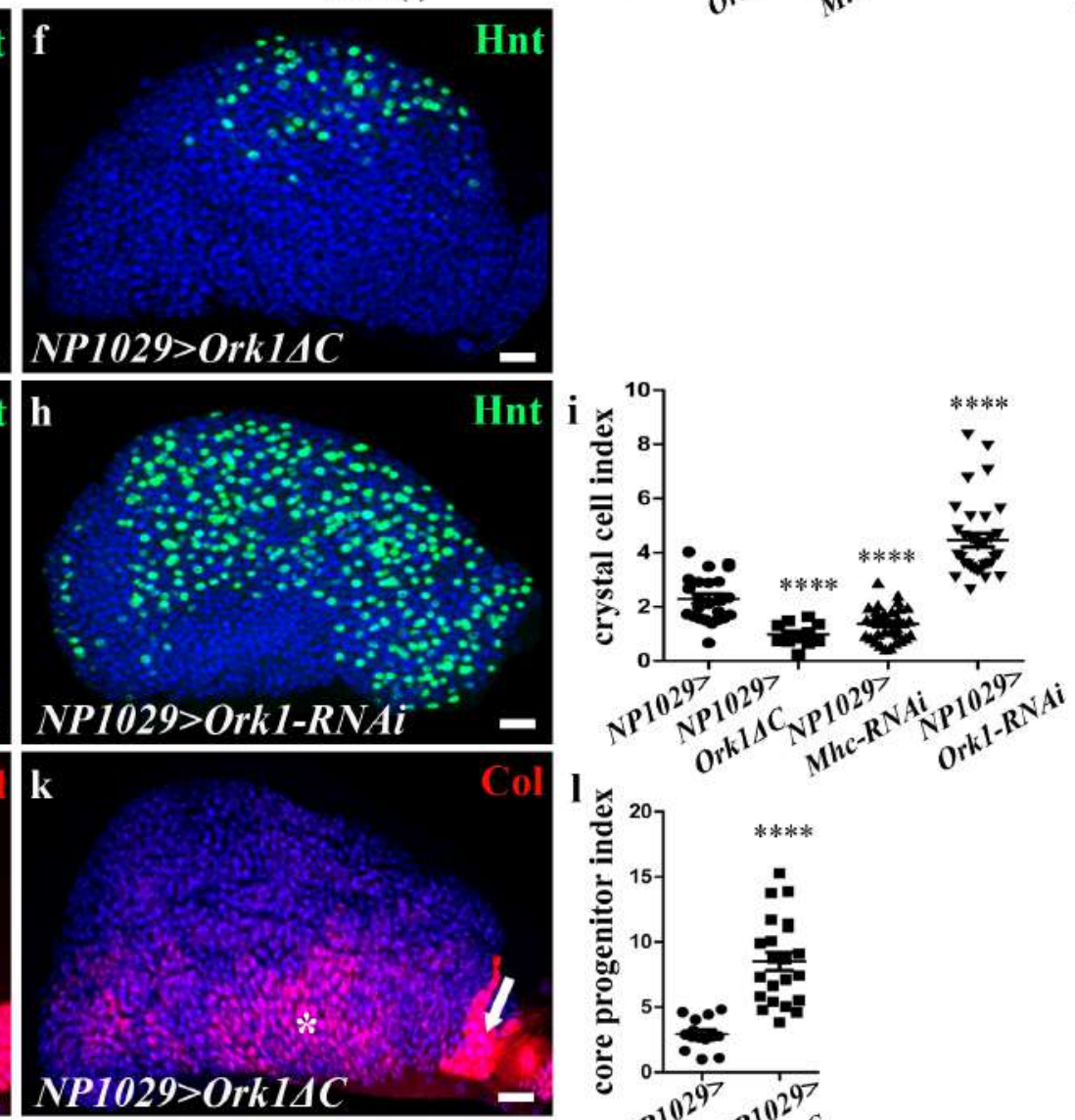

n

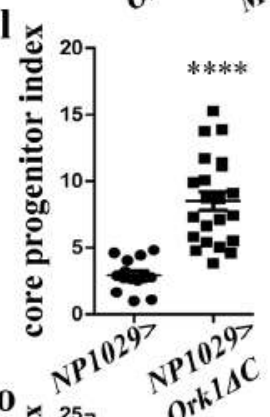

0
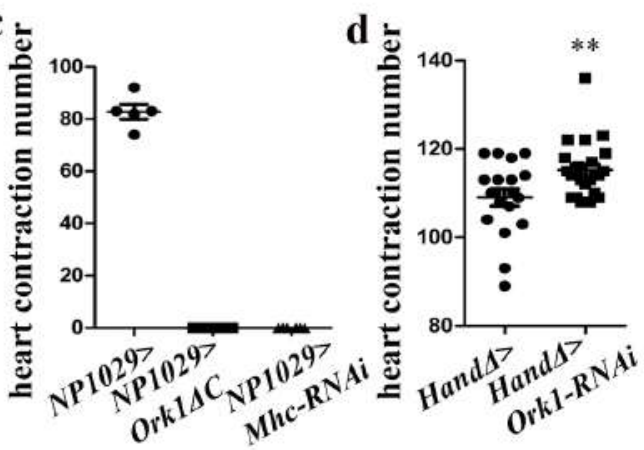

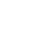




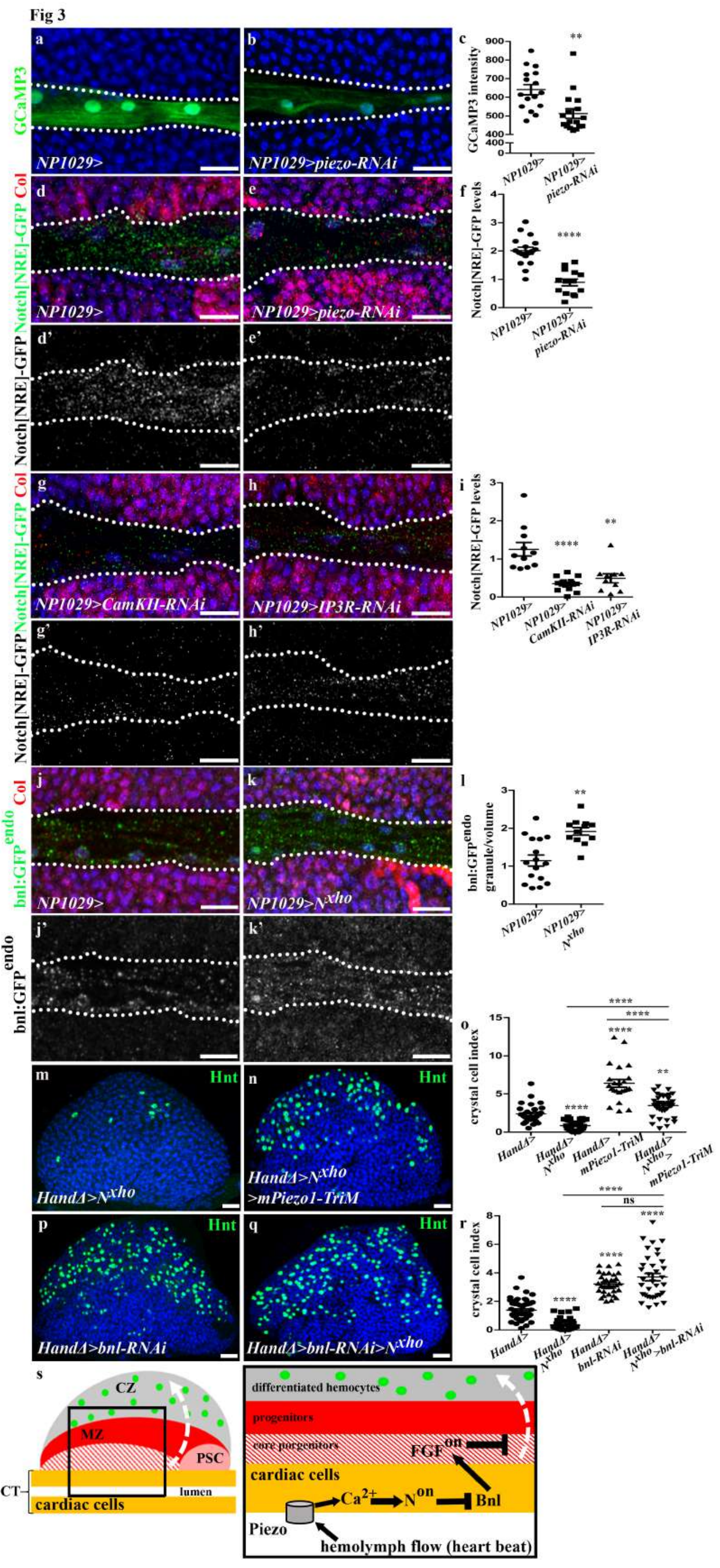


Fig 4
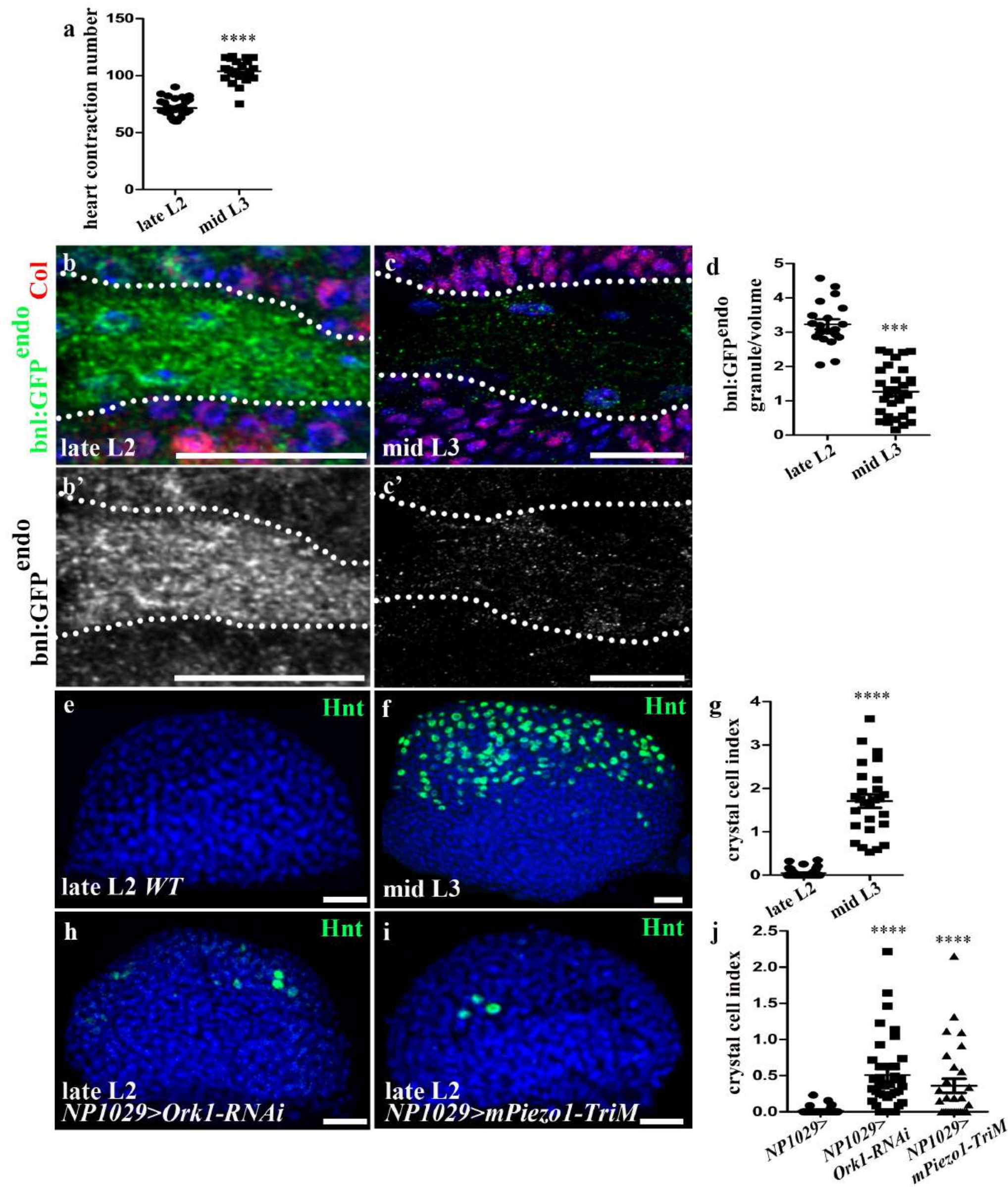
Sup Fig 1

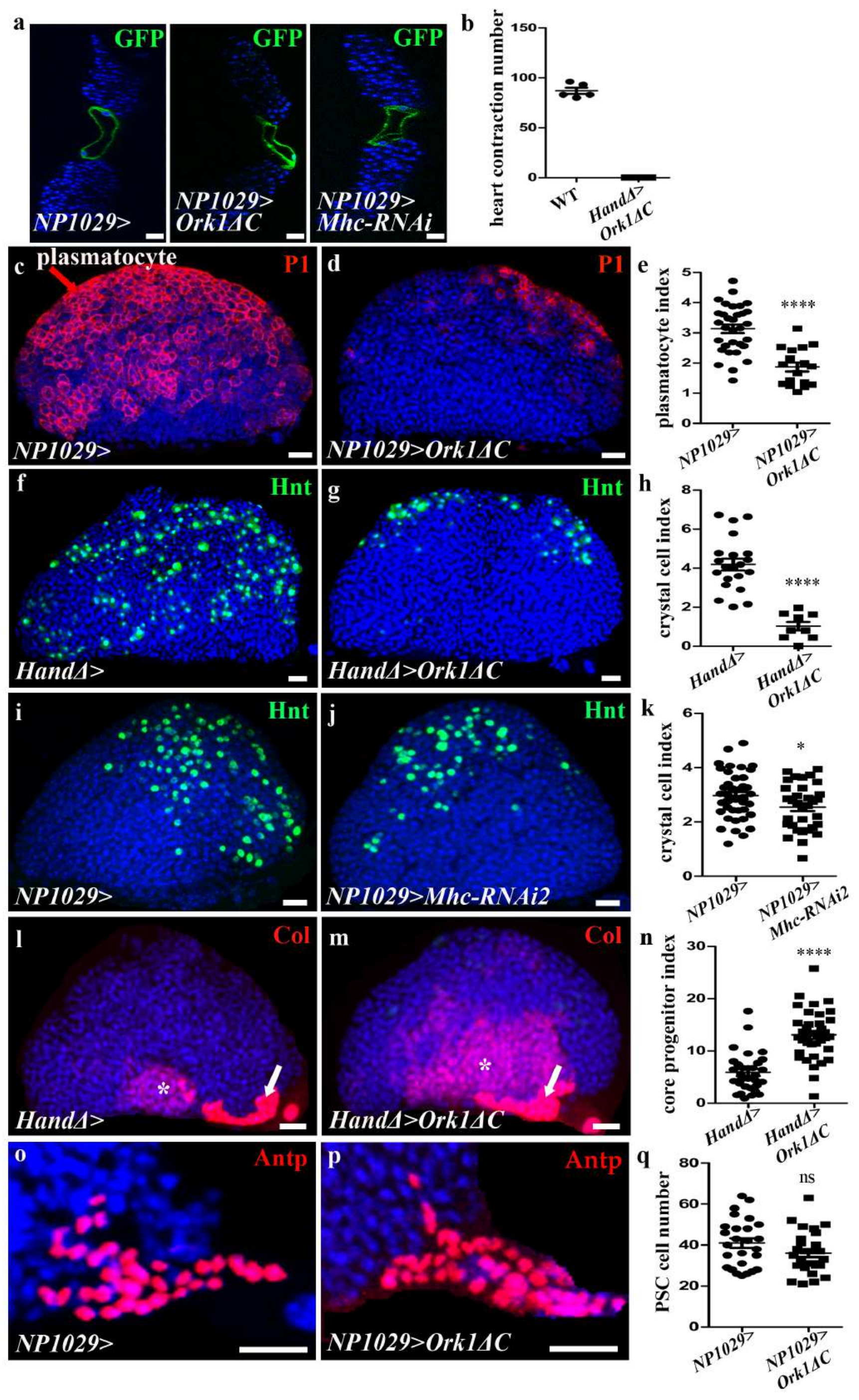


Sup Fig 2

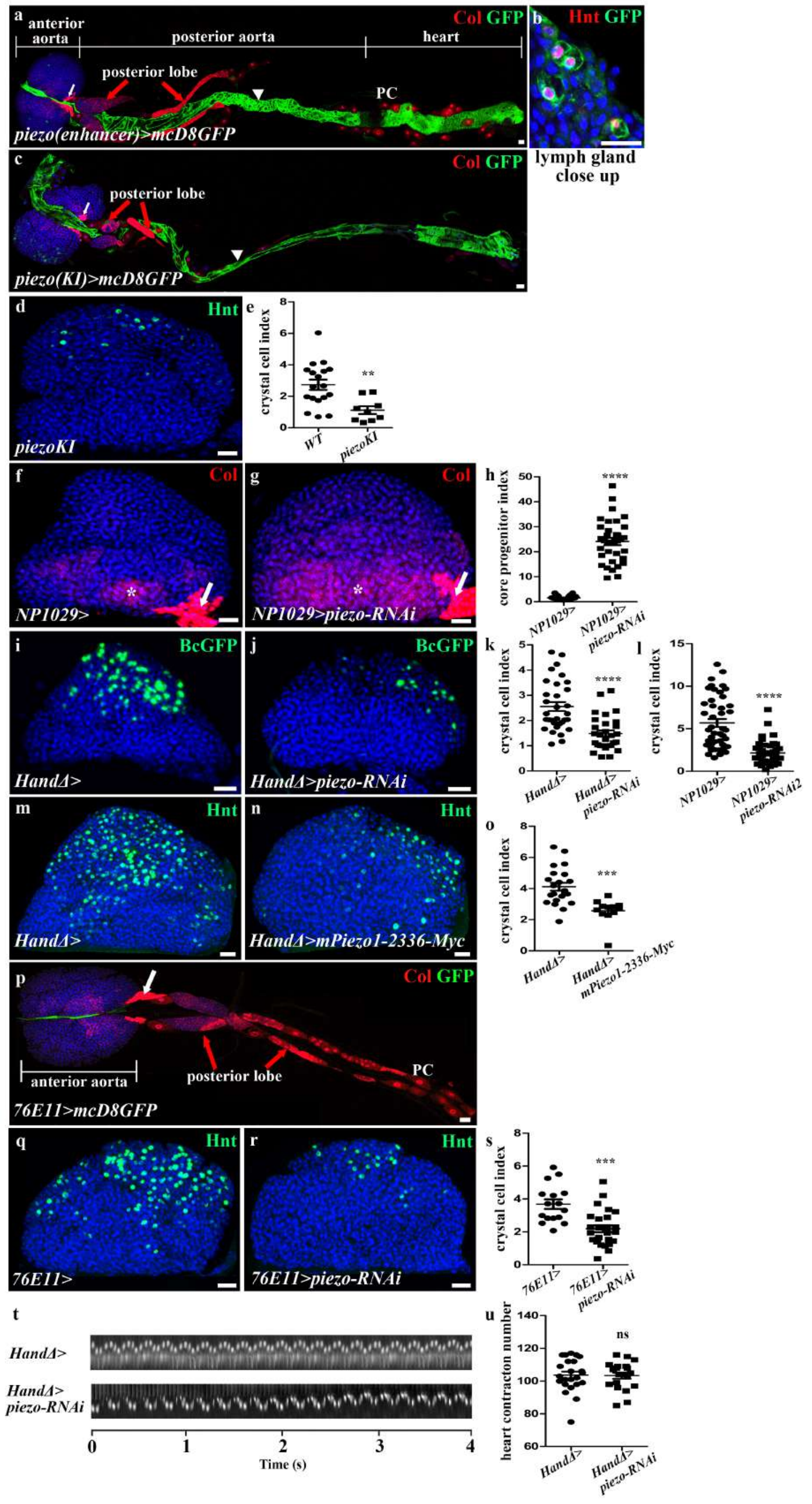


Sup Fig 3

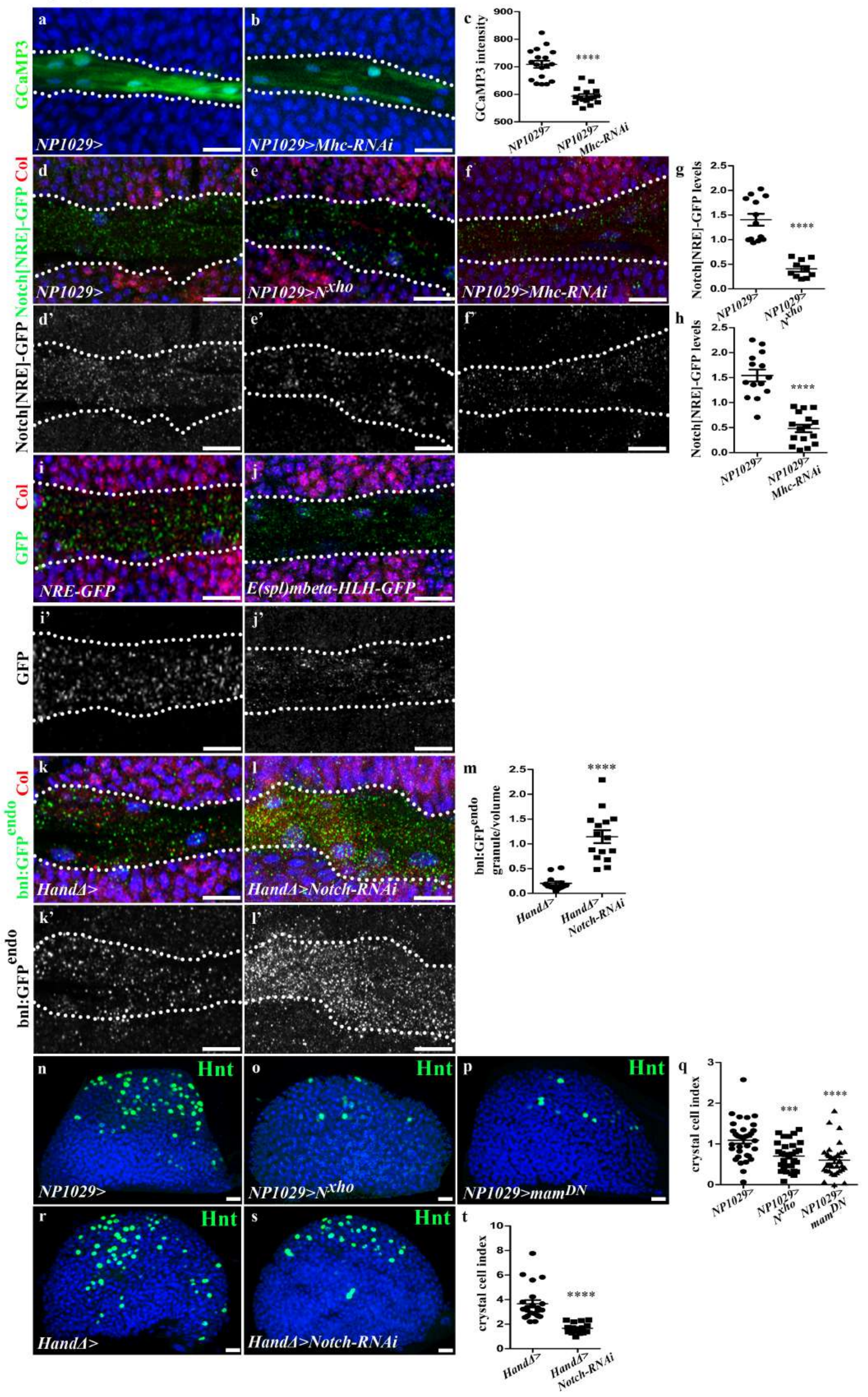




\section{Supplementary Files}

This is a list of supplementary files associated with this preprint. Click to download.

- abm8904SupplementaryMoviemov1seq1v1.avi

- abm8904SupplementaryMoviemov2seq2v1.avi

- abm8904SupplementaryMoviemov3seq3v1.avi

- abm8904SupplementaryMoviemov4seq4v1.avi

- abm8904SupplementaryMoviemov5seq5v1.avi

- abm8904SupplementaryMoviemov6seq6v1.avi

- abm8904SupplementaryMoviemov7seq7v1.avi

- abm8904SupplementaryMoviemov8seq8v1.avi 